Luis A. Florit · Wing San Hui · Fangyang Zheng

\title{
On real Kähler Euclidean submanifolds with non-negative Ricci curvature
}

Received November 13, 2003 and in revised form May 14, 2004

\begin{abstract}
We show that any real Kähler Euclidean submanifold $f: M^{2 n} \rightarrow \mathbb{R}^{2 n+p}$ with either non-negative Ricci curvature or non-negative holomorphic sectional curvature has index of relative nullity greater than or equal to $2 n-2 p$. Moreover, if equality holds everywhere, then the submanifold must be a product of Euclidean hypersurfaces almost everywhere, and the splitting is global provided that $M^{2 n}$ is complete. In particular, we conclude that the only real Kähler submanifolds $M^{2 n}$ in $\mathbb{R}^{3 n}$ that have either positive Ricci curvature or positive holomorphic sectional curvature are products of $n$ orientable surfaces in $\mathbb{R}^{3}$ with positive Gaussian curvature. Further applications of our main result are also given.
\end{abstract}

Keywords. Real Kahler submanifolds, non-negative Ricci curvature, non-negative holomorphic curvature, relative nullity

\section{Introduction}

Splitting theorems have always had a central role in Riemannian geometry, and in submanifolds theory in particular. A well known example is Hartman's cylinder theorem $([\overline{\mathrm{H}}])$ which is the extrinsic version of Cheeger-Gromoll's splitting theorem for complete manifolds of non-negative Ricci curvature.

In [F2, FZ1, FZ2, Z] we gave some splitting results for Euclidean submanifolds of non-positive sectional curvature. In [FZ1], we showed that, if $f: M^{m} \rightarrow \mathbb{R}^{m+p}$, $2 p \leq m$, is an isometric immersion in Euclidean space of an $m$-dimensional (connected) Riemannian manifold $M^{m}$ with non-positive sectional curvature and negative Ricci curvature, then $2 p=m$ and $f$ splits locally as a product of $p$ surfaces in $\mathbb{R}^{3}$. We say that an isometric immersion $f: M^{m} \rightarrow \mathbb{R}^{m+p}$ splits locally as a product of hypersurfaces if, for

Research of L. A. Florit partially supported by CNPq.

Research of F. Zheng partially supported by a NSF grant.

L. A. Florit: IMPA, Estrada Dona Castorina 110, 22460-320, Rio de Janeiro, Brazil; e-mail: luis@impa.br.

W. S. Hui, F. Zheng: The Ohio State University, Columbus, OH 43210, USA; e-mails:wshui@math.ohio-state.edu and zheng@math.ohio-state.edu.

Mathematics Subject Classification (2000): Primary 53B25; Secondary 53C40 
any $x \in M^{m}$, there exists a neighborhood $x \in U \subseteq M^{m}$, and, for each $1 \leq i \leq p$, a Riemannian manifold $U_{i}^{m_{i}}$ of dimension $m_{i}$ and an isometric immersion $f_{i}: \bar{U}_{i}^{m_{i}} \rightarrow \mathbb{R}^{m_{i}+1}$, such that

$$
U=U_{1} \times \cdots \times U_{p} \text { and }\left.f\right|_{U}=f_{1} \times \cdots \times f_{p} .
$$

There is no such general splitting result for positive sectional curvature, as the standard immersion of the unit sphere as a Euclidean hypersurface shows.

One of the main goals of this article is to study this kind of splitting when the Riemannian manifold has a Kähler structure. In this case we are able to give an analogous result to the aforementioned one in [FZ1], but with the reversed sign on the curvature, and even with weaker curvatures than the sectional one:

Theorem 1. Let $f: M^{2 n} \rightarrow \mathbb{R}^{2 n+p}$ be an isometric immersion of a Kähler manifold with $p \leq n$. Assume that $M^{2 n}$ has either positive Ricci curvature or positive holomorphic sectional curvature. Then $p=n$, and $f$ splits locally as a product of $n$ positively curved surfaces in $\mathbb{R}^{3}$. Moreover, the splitting is global if $M^{2 n}$ is complete.

As an immediate consequence of the above we deduce that there is no local isometric immersion of a Kähler manifold $M^{2 n}$ with positive sectional curvature into $\mathbb{R}^{2 n+p}, p \leq n$. In particular, no open subset of $\mathbb{C} \mathbb{P}^{n}$ admits an isometric immersion into $\mathbb{R}^{2 n+p}, p \leq n$.

Theorem 1 is a consequence of a more general result, where we study, for our situation, the index of relative nullity $v$ of the isometric immersion $f$, that is,

$$
v(x)=\operatorname{dim} \Delta(x),
$$

where $\Delta(x)=\operatorname{Ker} \alpha(x)=\left\{v \in T_{x} M: \alpha(v, w)=0, \forall w \in T_{x} M\right\}$ is the relative nullity of $f$ at $x$, i.e., the nullity space of the second fundamental form $\alpha$ of $f$ at $x$. It is well known that the positiveness of $v$ imposes quite strong restrictions on both $f$ and $M$, since, on any open subset where $v$ is constant, the relative nullity is a smooth integrable distribution with totally geodesic leaves in both $M$ and the ambient Euclidean space. Therefore, any lower bound on $v$ gives deep information on the submanifold. In particular, our aforementioned splitting results for Euclidean submanifolds of non-positive sectional curvature rely on a careful analysis of the relative nullity.

With this in mind, $[\overrightarrow{F 1}]$ was devoted to show that the index of relative nullity of a Euclidean submanifold $f: M^{m} \rightarrow \mathbb{R}^{m+p}$ with non-positive sectional curvature satisfies

$$
v \geq m-2 p,
$$

while the main result in [FZ1] is that, if equality holds, the submanifold should split locally as a product of $p$ hypersurfaces almost everywhere. Although it is easy to see that the same estimate on $v$ holds for any real Kähler Euclidean hypersurface $(p=1)$, even for codimension $p=2$ we can have $v \equiv 0$ for any $n$, as shown, for example, by the holomorphic complex hypersurface in $\mathbb{C}^{n+1}=\mathbb{R}^{2 n+2}$ given by $z_{n+1}=z_{1}^{2}+\cdots+z_{n}^{2}$. However, our next result states that the same estimate as in [F1] and the same splitting as in [FZ1] hold for Kähler Euclidean submanifolds, but with the reversed sign on weaker curvatures: 
Theorem 2. Let $f: M^{2 n} \rightarrow \mathbb{R}^{2 n+p}$ be an isometric immersion of a Kähler manifold with either non-negative Ricci curvature or non-negative holomorphic sectional curvature. Then the index of relative nullity $v$ of $f$ satisfies $v \geq 2 n-2 p$. Moreover, if $v \equiv 2 n-2 p$, then there is an open dense subset $W \subset M^{2 n}$ such that $\left.f\right|_{W}$ splits locally as a product of $p$ nowhere flat real Kähler Euclidean hypersurfaces with non-negative Ricci curvature.

Therefore, every such real Kähler Euclidean submanifold should be foliated almost everywhere by (open subsets of) affine Euclidean subspaces of dimension at least $2(n-p)$. Moreover, its curvature (and hence Ricci) tensor also has nullity of dimension $\mu \geq$ $2(n-p)$ since, by the Gauss equation, the relative nullity is always contained in that nullity. Thus, setting $\mu_{M}=\min _{x \in M} \mu$, which is an even integer number if $M^{2 n}$ is Kähler, we can state an immediate corollary with purely intrinsic assumptions:

Corollary 3. Let $M^{2 n}$ be a Kähler manifold with either non-negative Ricci curvature or non-negative holomorphic sectional curvature. Let $p=n-\mu_{M} / 2$. If $f: M^{2 n} \rightarrow \mathbb{R}^{2 n+p}$ is an isometric immersion, then there is an open dense subset $W \subset M^{2 n}$ such that $\left.f\right|_{W}$ splits locally as a product of $p$ nowhere flat real Kähler Euclidean hypersurfaces.

Theorem 2 is sharp in the sense that there are locally irreducible isometric immersions satisfying its hypothesis but with $v \equiv 2 n-2 p+1$. To see this, just compose a product of $p-1$ nowhere flat real Kähler hypersurfaces with a generic local immersion of $\mathbb{R}^{2 n+p-1}$ into $\mathbb{R}^{2 n+p}$. It is an interesting question if this is the only way to construct such submanifolds, as is the case for non-positive sectional curvature; see [FZ2].

Since each hypersurface factor in the conclusion of Theorem 2 has constant relative nullity of codimension two, we conclude that any real Kähler Euclidean submanifold with either non-negative Ricci curvature or non-negative holomorphic sectional curvature that has minimal index of relative nullity $v \equiv 2 n-2 p$ can now be explicitly locally parametrized. This can be done by means of the Gauss parametrization in terms of pseudoholomorphic surfaces in the sphere in the sense of [C]; cf. [DG1, DG2, FZ1, FZ2].

There are some known cylinder theorems for complete real Kähler submanifolds $f$ : $M^{2 n} \rightarrow \mathbb{R}^{2 n+p}$. The aforementioned fact that $v \geq 2 n-2$ for $p=1$ was used in $[\mathrm{A}$ | to show that $f$ splits as a surface in $\mathbb{R}^{3}$ and a $\mathbb{C}^{n-1}$ factor if either $f$ is real analytic, or the scalar curvature of $M^{2 n}$ is negative or non-negative. In fact, it was shown in [DG3] that the same holds true if $v=2 n-2$ in a dense connected subset, regardless of the codimension. For other cylinder theorems for complete minimal real Kähler submanifolds, see [DR2, DG4].

As a consequence of Theorem 2 we are also able to give a cylinder theorem of global nature that generalizes the one in [ $[\mathrm{FW}]$.

Corollary 4. Let $f: M^{2 n} \rightarrow \mathbb{R}^{2 n+p}$ be an isometric immersion of a complete Kähler manifold with either non-negative Ricci curvature or non-negative holomorphic sectional curvature. Then $v \geq 2 n-2 p$, and equality holds everywhere if and only if

$$
M^{2 n}=M_{1}^{2} \times \cdots \times M_{p}^{2} \times \mathbb{C}^{n-p} \text { and } f=f_{1} \times \cdots \times f_{p} \times I
$$


split globally, where $f_{i}: M_{i}^{2} \rightarrow \mathbb{R}^{3}, 1 \leq i \leq p$, is a complete isometrically immersed oriented surface of positive Gaussian curvature, and $I: \mathbb{C}^{n-p} \rightarrow \mathbb{R}^{2 n-2 p}$ is the identity map.

An analogous result holds if we require only $v\left(x_{0}\right)=2 n-2 p$ at one point $x_{0} \in M^{2 n}$ and the metric of $M^{2 n}$ to be real analytic; see Remark 12 Observe that there is a large family of complete, irreducible, minimal but not holomorphic real Kähler submanifolds in codimension $p=2$. They must be holomorphically ruled for $n \geq 3$ (so, $v \equiv 2 n-4=$ $2 n-2 p$ ) and admit a Weierstrass-type representation; cf. [DG4]. On the other hand, any complete, not everywhere minimal, real analytic Kähler submanifold $f: M^{2 n} \rightarrow \mathbb{R}^{2 n+2}$ in codimension $p=2$ must split as $f=f_{1} \times I$, where $I: \mathbb{C}^{n-2} \rightarrow \mathbb{R}^{2 n-4}$ is the identity map, and $f_{1}: N^{4} \rightarrow \mathbb{R}^{6}$ is either a product of two surfaces in $\mathbb{R}^{3}$, or a cylinder over a surface in $\mathbb{R}^{4}$, or a composition of isometric immersions $f_{1}=i \circ\left(f_{2} \times I\right)$, where $f_{2}: L^{2} \rightarrow \mathbb{R}^{3}, I: \mathbb{C} \rightarrow \mathbb{R}^{2}$ and $i: U \subset \mathbb{R}^{5} \rightarrow \mathbb{R}^{6}$; see [FZ4]. Moreover, it was shown in [FZ3] that any complete real Kähler Euclidean hypersurface must be a cylinder over a surface in $\mathbb{R}^{3}$, a result that generalizes the aforementioned one in $[\mathrm{A}]$.

The main ingredient in the proof of Theorem 2 is the general Proposition 10 of independent interest. In fact, it also allows us to obtain the following generalization of several results, as $[\bar{R}]$ for Kähler hypersurfaces in the sphere $\mathbb{S}_{c}^{N}$ of constant sectional curvature $c$, Theorem 4 in [FT] and Corollary 5 in [F1] (see also Corollary 11] below):

Theorem 5. If $M^{2 n} \subset \mathbb{S}_{c}^{2 n+p}$ is a real Kähler submanifold of a sphere with $p<n$, then $p=n-1$ and $M^{2 n}$ is (an open subset of) a product of $n$ round spheres in $\mathbb{R}^{3}$. That is, $M^{2 n} \subseteq \mathbb{S}_{c_{1}}^{2} \times \cdots \times \mathbb{S}_{c_{n}}^{2} \subset \mathbb{S}_{c}^{3 n-1} \subset \mathbb{R}^{3 n}$, where $1 / c=1 / c_{1}+\cdots+1 / c_{n}$.

We point out that the other part of the main result in $[\bar{R}]$, which deals with Kähler hypersurfaces in hyperbolic space $\mathbb{H}^{2 n+1}$, does not admit a generalization along the lines of Theorem 5 , since the hyperbolic space has a totally umbilical $\mathbb{R}^{2 n+p-1} \subset \mathbb{H}^{2 n+p}$.

\section{The second fundamental form of a real Kähler Euclidean submanifold}

In this section, we shall study some aspects of the general behavior of the second fundamental form of a real Kähler Euclidean submanifold, especially its $(1,1)$ part.

Let us first fix some notations. From now on, $f: M^{2 n} \rightarrow \mathbb{R}^{2 n+p}$ will be an isometric immersion of the Kähler manifold $M^{2 n}$ into the Euclidean space. Fix a point $x \in M^{2 n}$. The second fundamental form $\alpha=\alpha(x)$ of $f$ at $x$ is the symmetric bilinear map

$$
\alpha: T_{x} M \times T_{x} M \rightarrow T_{x}^{\perp} M=N \cong \mathbb{R}^{p},
$$

where $T_{x} M \cong \mathbb{R}^{2 n}$ is the real tangent space of $M^{2 n}$ at $x$, and $\left(T_{x}^{\perp} M,\langle\rangle,\right)$ is the normal space of $f$ at $x$. Extend $\alpha$ bilinearly over $\mathbb{C}$, and still denote it by $\alpha$,

$$
\alpha:\left(T_{x} M\right) \otimes \mathbb{C} \times\left(T_{x} M\right) \otimes \mathbb{C} \rightarrow N \otimes \mathbb{C} .
$$


Let $V$ be the space of type $(1,0)$ tangent vectors at $x$, that is, $V$ is the complex subspace of $\left(T_{x} M\right) \otimes \mathbb{C}$ given by $V=\left\{v-i J v: v \in T_{x} M\right\}$. Then $\left(T_{x} M\right) \otimes \mathbb{C} \cong V \oplus \bar{V}$. Write

$$
H=\left.\alpha\right|_{V \times \bar{V}} \quad \text { and } \quad S=\left.\alpha\right|_{V \times V}
$$

for the $(1,1)$ and $(2,0)$ parts of $\alpha$, respectively. Then $S: V \times V \rightarrow N \otimes \mathbb{C}$ is a symmetric complex bilinear map, while $H: V \times \bar{V} \rightarrow N \otimes \mathbb{C}$ is a Hermitian bilinear map, that is,

$$
H(Y, \bar{X})=\overline{H(X, \bar{Y})}, \quad \forall X, Y \in V .
$$

For simplicity, we will always write $S_{X Y}$ for $S(X, Y)$ and $H_{X \bar{Y}}$ for $H(X, \bar{Y})$.

Let us also extend the inner product $\langle$,$\rangle on N$ bilinearly over $\mathbb{C}$ to $N \otimes \mathbb{C}$, and still denote it by $\langle$,$\rangle . The Riemannian curvature tensor R$ of $M^{2 n}$ and the second fundamental form $\alpha$ are related by the Gauss equation:

$$
R_{A B C D}=\langle\alpha(A, D), \alpha(B, C)\rangle-\langle\alpha(A, C), \alpha(B, D)\rangle,
$$

for any real tangent vectors $A, B, C, D$ in $T_{x} M$. By our linear extension over $\mathbb{C}$, this equality also holds true for any $A, B, C, D$ in $\left(T_{X} M\right) \otimes \mathbb{C}=V \oplus \bar{V}$. Since $M^{2 n}$ is Kähler, we have $R_{X Y * *}=0$ if both $X$ and $Y$ are in $V$. Therefore, we get the following.

Proposition 6. Let $f: M^{2 n} \rightarrow \mathbb{R}^{2 n+p}$ be a real Kähler Euclidean submanifold, and consider $V, H, S$ as above. Then for any vectors $X, Y, Z, W$ in $V$,

$$
\begin{aligned}
\left\langle H_{X \bar{W}}, H_{Y \bar{Z}}\right\rangle & =\left\langle H_{Y \bar{W}}, H_{X \bar{Z}}\right\rangle, \\
\left\langle H_{X \bar{W}}, S_{Y Z}\right\rangle & =\left\langle H_{Y \bar{W}}, S_{X Z}\right\rangle, \\
\left\langle S_{X W}, S_{Y Z}\right\rangle & =\left\langle S_{X Z}, S_{Y W}\right\rangle, \\
R_{X \bar{Y} Z \bar{W}} & =\left\langle H_{X \bar{W}}, H_{Z \bar{Y}}\right\rangle-\left\langle S_{X Z}, \bar{S}_{Y W}\right\rangle .
\end{aligned}
$$

In particular, if $X=(v-i J v) / \sqrt{2}, Y=(w-i J w) / \sqrt{2}$ are unit vectors in $V$, we get

$$
\begin{aligned}
& R_{v J v J w w}=R_{X \bar{X} Y \bar{Y}}=\left|H_{X \bar{Y}}\right|^{2}-\left|S_{X Y}\right|^{2}, \\
& K(v, J v)=R_{X \bar{X} X \bar{X}}=\left|H_{X \bar{X}}\right|^{2}-\left|S_{X X}\right|^{2},
\end{aligned}
$$

where $K$ is the sectional curvature of $M^{2 n}$.

Define the index of pluriharmonic nullity $v_{J}=v_{J}(x)$ of $f$ at $x \in M^{2 n}$ by

$$
v_{J}=\operatorname{dim}_{\mathbb{C}} \Delta_{1,1}, \quad \text { where } \quad \Delta_{1,1}=\Delta_{1,1}(x):=\left\{X \in V: H_{X \bar{Y}}=0, \forall Y \in V\right\} .
$$

Our goal in this section is to prove the next general result.

Lemma 7. Let $f: M^{2 n} \rightarrow \mathbb{R}^{2 n+p}$ be a real Kähler Euclidean submanifold, $p \geq 1$. Take $x \in M^{2 n}$, and let $V, N, H, S$ be as above. Then $v_{J}(x) \geq n-p$. Moreover, if $v_{J}(x)=n-p$, there exists a basis $\left\{e_{1}, \ldots, e_{n}\right\}$ of $V$ such that $H_{e_{i} \bar{e}_{j}}=S_{e_{i} e_{j}}=0$ if either $i \neq j$ or $i=j>p$. Moreover, for $1 \leq i \leq p, H_{e_{i} \bar{e}_{i}} \neq 0, S_{e_{i} e_{i}}$ is collinear to $w_{i}=H_{e_{i} \bar{e}_{i}} /\left|H_{e_{i} \bar{e}_{i}}\right|$, and $\left\{w_{1}, \ldots, w_{p}\right\}$ is an orthonormal basis of the normal space $N$ at $x$.

Proof. We will prove this lemma by a series of claims. Set $q=v_{J}(x)$ and $V_{0}=\Delta_{1,1}(x)$. 
Claim 1. We have $H_{X \bar{X}} \neq 0$ for any $0 \neq X \in V_{0}^{\perp}$.

Proof. If not, then for any $Y \in V$, by $\left[1\right.$ we have $\left|H_{X \bar{Y}}\right|^{2}=\left\langle H_{X \bar{X}}, H_{Y \bar{Y}}\right\rangle=0$. So $X \in V_{0}$, which is a contradiction.

Claim 2. If $n \geq 2$, then there exist non-zero vectors $X, Y \in V_{0}^{\perp}$ such that $H_{X \bar{Y}}=0$.

Proof. Let $\left\{e_{1}, \ldots, e_{n-q}\right\}$ be a basis of $V_{0}^{\perp}$. In the following, we will write $H_{i}$ for $H_{e_{i} \bar{e}_{j}}$. Consider the vectors $H_{1 \overline{1}}, H_{1 \overline{2}}, \ldots, H_{1 \overline{n-q}}$ in $N \otimes \mathbb{C} \cong \mathbb{C}^{p}$. By assumption, we have $p \leq n-q$. If $p<n-q$, then these vectors are linearly dependent. So there is $Y \neq 0$ in $V$ such that $H_{e_{1} \bar{Y}}=0$, and Claim 2 is proved. Now consider the case $p=n-q$. We may also assume that the sets $\left\{H_{1 \overline{1}}, H_{12}, \ldots, H_{1 \bar{p}}\right\}$ and $\left\{H_{2 \overline{1}}, H_{2 \overline{2}}, \ldots, H_{2 \bar{p}}\right\}$ are each linearly independent in $N \otimes \mathbb{C}$. Both sets are bases of $\mathbb{C}^{p}$, so there is a non-degenerate complex $p \times p$ matrix $B=\left(B_{i j}\right)$ such that

$$
H_{1 \bar{i}}=\sum_{j=1}^{p} B_{i j} H_{2 \bar{j}}
$$

for each $1 \leq i \leq p$. Let $X=e_{1}-\lambda e_{2}$, and $Y=\sum y_{i} e_{i}$. Then we have

$$
H_{X \bar{Y}}=\sum \bar{y}_{i} H_{1 \bar{i}}-\lambda \sum \bar{y}_{i} H_{2 \bar{i}}=\sum_{j}\left\{\sum_{i} \bar{y}_{i}(B-\lambda I)_{i j}\right\} H_{2 \bar{j}}
$$

Thus, if we choose $\lambda$ to be an eigenvalue of the matrix $B$, and $\left(\bar{y}_{1}, \ldots, \bar{y}_{p}\right) \neq 0$ the corresponding eigenvector, then $H_{X \bar{Y}}=0$. This completes the proof of Claim 2.

Next, for each $X \in V_{0}^{\perp}$, denote by $H_{X}: V_{0}^{\perp} \rightarrow N \otimes \mathbb{C}$ the linear map given by $H_{X}(Y)=H(Y, \bar{X})$, and let $K(X)=\operatorname{Ker} H_{X}$. We will call the subspace of $N \otimes \mathbb{C}$ spanned by $H_{X \bar{Y}}$ for all $X, Y$ in $V_{0}^{\perp}$ the image space of $H$.

Claim 3. We have $p=n-q$, the image space of $H$ coincides with $N \otimes \mathbb{C}$, and there exists $0 \neq X \in V_{0}^{\perp}$ such that $K(X)$ is $(n-q-1)$-dimensional.

Proof. We will perform induction on $n-q$. When $n-q=1, p$ must be $1, H_{X}$ has trivial kernel for any non-zero $X \in V_{0}^{\perp}$, and the image space of $H$ is $N \otimes \mathbb{C}$ by Claim 1 .

Now assume that Claim 3 is true when restricted to any proper subspace of $V_{0}^{\perp}$. For our $V_{0}^{\perp}$ of dimension $n-q \geq 2$, fix $0 \neq X \in V_{0}^{\perp}$. From Claims 1 and 2 we see that $\operatorname{dim} K(X)=n-q-r$ for some $1 \leq r<n-q$.

Define $P=\operatorname{Im}\left(H_{X}\right) \subset N \otimes \mathbb{C}$. It has complex dimension $r$. Consider the subspace $Q=P+\bar{P} \subseteq N \otimes \mathbb{C}$, and denote its complex dimension by $s \geq r$. Since $\bar{Q}=Q$, we know that $Q=N_{0} \otimes \mathbb{C}$ for some real linear subspace $N_{0} \subseteq N$ of real dimension $s$. Let $N_{1}$ be the orthogonal complement of $N_{0}$ in $N$, and write $V_{1}=K(X)$. Then for any $Y, Z$ in $V_{1}$ and any $W \in V$, by (1) we have

$$
\left\langle H_{Y \bar{Z}}, H_{X \bar{W}}\right\rangle=\left\langle H_{Y \bar{W}}, H_{X \bar{Z}}\right\rangle=0,
$$


and similarly, $\left\langle H_{Y \bar{Z}}, H_{W \bar{X}}\right\rangle=0$. That is, $H_{Y \bar{Z}}$ is perpendicular to $Q$, or, in other words, the restriction $\left.H\right|_{V_{1} \times \bar{V}_{1}}$ has its image contained in $N_{1} \otimes \mathbb{C}$. Note that the dimension of $N_{1}$ is $p-s$, which is less than or equal to $n-q-r$, the dimension of $V_{1}$. So by the inductive hypothesis, we know that $p-s=n-q-r$, which implies that $p=n-q$ and $r=s$, and that there exists $0 \neq Y \in V_{1}$ such that $\operatorname{Ker}\left(\left.H_{Y}\right|_{V_{1}}\right) \subset V_{1}$ has codimension 1 . Moreover, the image space of $\left.H\right|_{V_{1} \times \bar{V}_{1}}$ is equal to $N_{1} \otimes \mathbb{C}$, which implies that the image space of $H$ is equal to $N \otimes \mathbb{C}$.

We claim that $K(Y) \subset V_{0}^{\perp}$ has codimension 1, which completes the proof of Claim 3 . Since $H_{Y \bar{Y}} \neq 0$, it suffices to show that, for any $Z \in V_{0}^{\perp}$, we have $W=Z-\lambda Y \in K(Y)$, where $\lambda=\lambda(Z)=\left\langle H_{Z \bar{Y}}, H_{Y \bar{Y}}\right\rangle /\left|H_{Y \bar{Y}}\right|^{2}$. Observe that $\left\langle H_{W \bar{Y}}, H_{Y \bar{Y}}\right\rangle=0$.

First, write $m=n-q-r$, and choose a basis $\left\{e_{1}, \ldots, e_{m}\right\}$ of $V_{1}$ such that $Y=e_{1}$ and $\operatorname{Ker}\left(\left.H_{Y}\right|_{V_{1}}\right)=\operatorname{span}\left\{e_{2}, \ldots, e_{m}\right\}$. By the inductive hypothesis, the space $N_{1} \otimes \mathbb{C}$ is spanned by $H_{i} \bar{j}$ for $1 \leq i, j \leq m$. By (1), we have

$$
\left\langle H_{W \bar{Y}}, H_{i \bar{j}}\right\rangle=\left\langle H_{W \overline{e_{j}}}, H_{e_{i} \bar{Y}}\right\rangle,
$$

which is zero if $i>1$, or if $i=1$ and $j>1$ since $H_{1 \bar{j}}=0$ in this case. We also know that the left hand side is zero if $i=j=1$, by our construction of $W$. So $H_{W \bar{Y}}$ is orthogonal to $N_{1} \otimes \mathbb{C}$, that is, $H_{W \bar{Y}} \in Q$.

Secondly, from the fact that $r=s$, we know that $Q=\operatorname{Im}\left(H_{X}\right)=H(X, \bar{V})$. But $\left\langle H_{W \bar{Y}}, H_{X \bar{U}}\right\rangle=\left\langle H_{W \bar{U}}, H_{X \bar{Y}}\right\rangle=0$ for all $U \in V$ since $H_{X \bar{Y}}=0$. We conclude that $H_{W \bar{Y}}=0$ as desired.

Now we are ready to finish the proof of Lemma 7 . By Claim 3, there exists $e_{1} \in V_{0}^{\perp}$ such that the kernel $K\left(e_{1}\right)$ is $(n-q-1)$-dimensional. Note that $e_{1} \notin K\left(e_{1}\right)$. Applying Claim 3 to the restriction of $H$ to $K\left(e_{1}\right) \times \overline{K\left(e_{1}\right)}$, we find $e_{2} \in K\left(e_{1}\right)$ for which $H_{e_{2}}$ has codimension one kernel in $K\left(e_{1}\right)$. Inductively, we get a basis $\mathcal{B}=\left\{e_{1}, \ldots, e_{p}\right\}$ of $V_{0}^{\perp}$ such that $H_{i \bar{j}}=0$ whenever $i \neq j$. This fact and (1) also imply that $0 \neq H_{i \bar{i}} \perp H_{j \bar{j}}$ for any $i \neq j$, and we get the desired orthonormal basis $\left\{w_{1}, \ldots, w_{p}\right\}$ of $N$. Extend $\mathcal{B}$ to a basis $\mathcal{B}^{\prime}=\left\{e_{1}, \ldots, e_{n}\right\}$ of $V$ such that $\left\{e_{p+1}, \ldots, e_{n}\right\}$ is a basis of $V_{0}$

To see that $S$ is also diagonal under $\mathcal{B}^{\prime}$, fix any $i \neq j$. For any $1 \leq k \leq p$, we see by (2) that $\left\langle H_{k \bar{k}}, S_{i j}\right\rangle=\left\langle H_{i \bar{k}}, S_{k j}\right\rangle=\left\langle H_{j \bar{k}}, S_{k i}\right\rangle$. So it is zero if $k \neq i$ or $k \neq j$, which always happens as $i \neq j$. That is, $S_{i j}$ is perpendicular to $w_{k}$ for all $k$, so it must be zero. Similarly, $S_{i i}=0$ if $i>p$.

The formula (2) also implies that $S_{i i} \perp H_{j \bar{j}}$ for any $j \neq i$, thus $S_{i i}$ points in the direction of $w_{i}$, for each $1 \leq i \leq p$. This completes the proof of Lemma 7

Remark 8. From its very definition, we see that the vanishing of the $(1,1)$ part $H$ of the second fundamental form of $f$ is equivalent to $f$ being pluriharmonic (also called circular $)$, that is, $\alpha(J X, Y)=\alpha(X, J Y)$ for all $X, Y \in T M$. Therefore, Lemma 7] says that the second fundamental form of $f$ is diagonal if the immersion is "as far as possible" from being pluriharmonic. It was shown in Theorem 1.2 of [DR1] that a Euclidean Kähler submanifold is pluriharmonic if and only if it is minimal. We point out that this result follows immediately from (1), since the mean curvature $h$ of the immersion can be written as $h=\sum_{i=1}^{n} H_{i \bar{i}}$ and then $\|h\|^{2}=\sum_{i, j}\left\langle H_{i \bar{i}}, H_{j \bar{j}}\right\rangle=\sum_{i, j}\left\|H_{i \bar{j}}\right\|^{2}$. 


\section{The main result and some consequences}

With the notations of Lemma 7, assume that $v_{J}(x)=n-p$ for all $x \in M^{2 n}$. Then $v \geq 2 n-2 p$ and there exists a tangent diagonalizing frame $\left\{e_{1}, \ldots, e_{p}\right\}$ of type $(1,0)$ vectors in $\Delta_{1,0}^{\perp}=V \cap\left(\Delta^{\perp} \otimes \mathbb{C}\right)$ at each $x$. Note that this frame is unique up to permutation and scalings. In other words, the set $\left\{\left[e_{1}\right], \ldots,\left[e_{p}\right]\right\}$ is unique in the symmetric power $S^{n}\left(\mathbb{P}\left(\Delta_{1,0}^{\perp}\right)\right)$ of the projectivized holomorphic $\Delta_{1,0}^{\perp}$ bundle. Hence, since $H$ is smooth, in a sufficiently small neighborhood $U$ of $x$, we can take a smooth frame $\left\{e_{1}, \ldots, e_{n}\right\}$ that has the diagonalization property of Lemma 7 at each point in $U$. In particular, we also obtain from Lemma 7 a smooth orthonormal normal frame $\left\{w_{1}, \ldots, w_{p}\right\}$.

Assume further that $v \equiv 2 n-2 p$. For each $1 \leq i \leq p$, consider the shape tensor $A_{w_{i}}$ on $M^{2 n}$ defined by $\left\langle A_{w_{i}} X, Y\right\rangle=\left\langle\alpha_{f}(X, Y), w_{i}\right\rangle$, and let

$$
V_{i}=\operatorname{Im} A_{w_{i}} .
$$

Thus, the set $\left\{\operatorname{Re} e_{1}, \operatorname{Im} e_{1}, \ldots, \operatorname{Re} e_{i-1}, \operatorname{Im} e_{i-1}, \operatorname{Re} e_{i+1}, \operatorname{Im} e_{i+1}, \ldots, \operatorname{Re} e_{p}, \operatorname{Im} e_{p}\right\}$ is a base of $V_{i}^{\perp} \cap \Delta^{\perp}$ by Lemma 7, and each $V_{i}$ is a two-dimensional complex smooth distribution on $M^{2 n}$ such that

$$
V_{1} \oplus \cdots \oplus V_{p}=\Delta^{\perp}
$$

Lemma 9. With the notations of Lemma 7, if $v \equiv 2 v_{J} \equiv 2 n-2 p$, then the normal bundle of $f$ is flat. Moreover, each $w_{i}$ is parallel in the normal connection, the decomposition (8) is orthogonal and both $V_{i} \oplus \Delta$ and $V_{i}^{\perp}$ are integrable, for all $1 \leq i \leq p$.

Proof. Let $\psi_{i j}$ be the 1 -forms defined by $\psi_{i j}(X)=\left\langle\nabla_{X}^{\perp} w_{i}, w_{j}\right\rangle$. To show that each $w_{i}$ is parallel it suffices to see that $\psi_{i j}=0$ for all $i, j$. Recall that the Codazzi equation for $A_{w_{i}}$ is

$$
\nabla_{X}\left(A_{w_{i}} Y\right)-A_{w_{i}} \nabla_{X} Y-A_{\nabla_{X} w_{i}} Y=\nabla_{Y}\left(A_{w_{i}} X\right)-A_{w_{i}} \nabla_{Y} X-A_{\nabla_{Y} w_{i}} X .
$$

Taking $X, Y \in V_{i}^{\perp}=\operatorname{Ker} A_{w_{i}}$ in 97 we easily infer using 8 , that

$$
A_{w_{j}}\left(\psi_{i j}(X) Y-\psi_{i j}(Y) X\right)=0, \quad \forall X, Y \in V_{i}^{\perp}, 1 \leq j \leq p .
$$

Suppose that there are $X_{0} \in V_{i}^{\perp}$ and $j \neq i$ such that $\psi_{i j}\left(X_{0}\right) \neq 0$. The above equation implies that $V_{i}^{\perp} \subset V_{j}^{\perp} \oplus \operatorname{span}\left\{X_{0}\right\}$, that is, $T_{x} M \neq V_{i}^{\perp}+V_{j}^{\perp}=\left(V_{i} \cap V_{j}\right)^{\perp}$, which is a contradiction by $(8)$. Thus $V_{i}^{\perp} \subset \operatorname{Ker} \psi_{i j}$ for all $i, j$. By the orthonormality of $\left\{w_{i}\right\}$ we have $\psi_{i j}=-\psi_{j i}$. Therefore, $T_{x} M=V_{i}^{\perp}+V_{j}^{\perp} \subset \operatorname{Ker} \psi_{i j}$.

The Ricci equation now implies that the $V_{i}$ 's are orthogonal, since it says that $\left[A_{w_{i}}, A_{w_{j}}\right]=0$. The integrability of $V_{i}^{\perp}$ follows from $[9]$ if we take $X, Y \in V_{i}^{\perp}$, since $w_{i}$ is parallel. This concludes our proof since $V_{i} \oplus \Delta=\bigcap_{j \neq i} V_{j}^{\perp}$.

Lemmas 7 and 9 are the principal ingredients to deduce the next main result, which is of interest in its own right:

Proposition 10. Let $f: M^{2 n} \rightarrow \mathbb{R}^{2 n+p}$ be any Kähler Euclidean submanifold, $p \leq n$. Then $v_{J} \geq n-p$. Moreover: 
(i) If $v_{J} \equiv n-p$, then $v \geq 2 n-2 p$.

(ii) If $v_{J} \equiv n-p$ and $v \equiv 2 n-2 p$, then there is an open dense subset $W \subset M^{2 n}$ such that $\left.f\right|_{W}$ splits locally as a product of $p$ nowhere flat (with relative nullity of codimension two) real Kähler Euclidean hypersurfaces.

(iii) If $v \equiv v_{J} \equiv 0$, then $f$ splits locally as a product of $p$ nowhere flat orientable surfaces in $\mathbb{R}^{3}$ on the whole $M^{2 n}$. This splitting is global if $M^{2 n}$ is complete.

Proof. (iii) We have $p=n$. Lemma 9 and the local de Rham decomposition theorem imply that the metric on $M^{2 n}$ splits locally. Replacing $U$ by a smaller simply connected neighborhood of $x$ if necessary, we get the isometric splitting $U=U_{1} \times \cdots \times U_{n}$ into factors of complex dimension one, with $T U_{i}=V_{i}$. Since by Lemmas 7 and 9 we have $\alpha\left(V_{i}, V_{j}\right)=0$ for all $1 \leq i \neq j \leq n$, the Main Lemma of [M] gives the splitting of $\left.f\right|_{U}$. The surfaces are nowhere flat by (7). The complete case follows easily from the global de Rham decomposition theorem upon lifting $f$ to the universal cover of $M^{2 n}$.

(ii) The proof follows just as above after constructing the factors with the same arguments of Lemma 5 in [F2], so we will skip it here. We only recall that the presence of the open dense subset $W$ is due to the fact that the relative nullity can indeed "jump" between the hypersurface factors.

The strength of this proposition becomes clear when we use it to easily derive the proofs of the theorems stated in the introduction:

Proof of Theorems 1 and 2. By Proposition 10, all we have to show is that $v \geq 2 v_{J}$. If the holomorphic sectional curvature is assumed to be non-negative, then for $X \in \Delta_{1,1}$,

$$
R_{X \bar{X} X \bar{X}}=\left|H_{X \bar{X}}\right|^{2}-\left|S_{X X}\right|^{2}=-\left|S_{X X}\right|^{2} \geq 0
$$

implies that $S_{X X}=0$, and by (3), $S_{X Y}=0$ for any $Y \in V$. If the Ricci curvature is assumed to be non-negative, then for any $X \in \Delta_{1,1}$, the Ricci curvature in the direction of $X$ is given by

$$
\sum_{i=1}^{n}\left(\left|H_{X \bar{v}_{i}}\right|^{2}-\left|S_{X v_{i}}\right|^{2}\right)=-\sum_{i=1}^{n}\left|S_{X v_{i}}\right|^{2}
$$

where $\left\{v_{i}\right\}_{i=1}^{n}$ is any unitary basis of $V$. So we know that $S_{X Y}=0$ for any $Y \in V$. Thus, under either curvature assumption, $\Delta_{1,1}$, and hence $\Delta_{1,1} \oplus \bar{\Delta}_{1,1}$, is contained in $\Delta \otimes \mathbb{C}$. Therefore, $2 v_{J} \leq v$ and the proof is complete.

Proof of Theorem 5. Consider the inclusion in Euclidean space $M^{2 n} \subset \mathbb{S}_{c}^{2 n+p} \subset \mathbb{R}^{2 n+p+1}$, and call it $f$. If $\eta$ is the Gauss map of the sphere, then for $f$ and $X \in V, X \neq 0$, we have $\left\langle H_{X \bar{X}}, \eta\right\rangle=\sqrt{c}\|X\|^{2} \neq 0$. So we obtain $v \equiv v_{J} \equiv 0 \leq n-p-1$. Proposition 10 (iii) then implies that $f\left(M^{2 n}\right)$ is locally contained in a product of $n=p+1$ surfaces in $\mathbb{R}^{3}$. Now, the only way that this product is included in $\mathbb{S}_{c}^{3 n-1}$ is if each factor itself is an open subset of a round two-sphere.

With similar arguments we prove the following: 
Corollary 11. Let $f: M^{2 n} \rightarrow \mathbb{R}^{2 n+p}$ be a real Kähler submanifold with $p \leq n$. Assume either that (a) $f\left(M^{2 n}\right)$ is contained in some strictly convex hypersurface of $\mathbb{R}^{2 n+p}$, or (b) $M^{2 n}$ is complete with sectional curvature bounded from below, $f\left(M^{2 n}\right)$ is bounded and $f$ is real analytic. Then $p=n$ and $f$ splits as a product of $n$ orientable surfaces in $\mathbb{R}^{3}$.

Proof. It is clear that in case (a) we have a positive definite shape operator, so the assertion follows as in Theorem 5. For part (b), take $h: M^{2 n} \rightarrow \mathbb{R}$ defined by $2 h(x)=$ $\langle f(x), f(x)\rangle$. By Omori's Lemma ([0]), there is $y \in M^{2 n}$ such that $\operatorname{Hess}_{h}(y)(X, X)<$ $\|X\|^{2}$ for all $X \in T_{y} M, X \neq 0$. But $\operatorname{Hess}_{h}(y)(X, X)=\|X\|^{2}+\langle\alpha(X, X), f(y)\rangle$. Hence, $A_{-f(y)^{\perp}}>0$.

Proof of Corollary 4 The relative nullity estimate follows from Theorem 2 Assume that the equality holds everywhere. From Theorem 2 we also infer that $M^{2 n}$ has nonnegative Ricci curvature with either curvature assumption. In fact, we easily see that the Ricci curvature restricted to $\Delta^{\perp}$ is positive. Since the leaves of the (complex) relative nullity distribution $\Delta$ are totally geodesic in both $M^{2 n}$ and $\mathbb{R}^{2 n+p}$, and they are complete whenever $M^{2 n}$ is complete, we deduce that $M^{2 n}$ contains $2 n-2 p$ linearly independent lines. So, by Hartman's theorem [H] (see Theorem 5.10 in [D] for the Ricci curvature version using the splitting theorem of Cheeger-Gromoll), $f$ has a complex factor, $M^{2 n}=$ $N^{2 p} \times \mathbb{C}^{n-p}$ and $f=f_{1} \times I$, where $f_{1}: N^{2 p} \rightarrow \mathbb{R}^{3 p}$ is an isometric immersion of a Kähler manifold with positive Ricci curvature and $I$ is the identity map. The corollary follows by applying Theorem 1 to $f_{1}$.

Remark 12. For an $f: M^{2 n} \rightarrow \mathbb{R}^{2 n+p}$ as in Corollary 4, let $r=\min v$. It is well known that the subset $U=v^{-1}(r) \subset M^{2 n}$ is open and the leaves of $\Delta$ restricted to $U$ are complete. Hence, again by Hartman's theorem, we have a splitting $M^{2 n}=N^{2 n-r} \times \mathbb{R}^{r}$ and $f=f_{1} \times I$, with $r \geq 2 n-2 p$. Thus, if we require the maximal Euclidean factor of $f$ to have dimension $2 n-2 p$ then, by Theorem 1 . $f_{1}$ also splits locally in $U \cap\left(N^{2 p} \times\{0\}\right)$ as a product of $p$ orientable surfaces in $\mathbb{R}^{3}$ of positive Gaussian curvature in a unique way. Therefore, if we further require the metric of $M^{2 n}$ to be real analytic, we easily obtain the same global splitting as in Corollary 4 although in this case the surface factors will have positive Gaussian curvature almost everywhere.

\section{References}

[A] Abe, K.: On a class of hypersurfaces of $\mathbb{R}^{2 n+1}$. Duke Math. J. 41, 865-874 (1974) Zbl 0304.53044 MR 50 \#3153

[C] Calabi, E.: Quelques applications de l'analyse complexe aux surfaces d'aire minimale. In: Topics in Complex Manifolds, Presses Univ. de Montréal, Montréal, 59-81 (1968)

[D] Dajczer, M. et al.: Submanifolds and Isometric Immersions. Math. Lec. Series 13, Publish or Perish, Houston (1990) Zbl 0705.53003 MR 92i:53049

[DG1] Dajczer, M., Gromoll, D.: Gauss parametrizations and rigidity aspects of submanifolds. J. Differential Geom. 22, 1-12 (1985) Zbl 0588.53007 MR 87g:53088a

[DG2] Dajczer, M., Gromoll, D.: Real Kaehler submanifolds and uniqueness of the Gauss map. J. Differential Geom. 22, 13-28 (1985) Zbl 0587.53051 MR 87g:53088b 
[DG3] Dajczer, M., Gromoll, D.: Rigidity of complete Euclidean hypersurfaces. J. Differential Geom. 31, 401-416(1990) Zbl 0667.53003 MR 91d:53083

[DG4] Dajczer, M., Gromoll, D.: The Weierstrass representation for complete minimal real Kaehler submanifolds of codimension two. Invent. Math. 119, 235-242 (1995) Zbl 0827.53047 MR 97k:58047

[DR1] Dajczer, M., Rodríguez, L.: Rigidity of real Kaehler submanifolds. Duke Math. J. 53, 211220 (1986) Zbl 599.53005 MR 87g:53089

[DR2] Dajczer, M., Rodríguez, L.: Complete real Kähler minimal submanifolds. J. Reine Angew. Math. 419, 1-8 (1991) Zbl 0726.53041 MR 92g:53057

[FT] Ferreira, M., Tribuzy, R.: Codimension two Kähler submanifolds in space forms. Arch. Math. (Basel) 79, 520-528 (2002) Zbl 01889282 MR 2004b:53104

[F1] Florit, L.: On submanifolds with nonpositive extrinsic curvature. Math. Ann. 298, 187-192 (1994) Zbl 0810.53011 MR 95c:53075

[F2] Florit, L.: A splitting theorem for euclidean submanifolds of nonpositive sectional curvature. Math. Z. 225, 685-690 (1997) Zbl 0916.53002 MR 98d:53079

[FZ1] Florit, L., Zheng, F.: On nonpositively curved euclidean submanifolds: splitting results. Comment. Math. Helv. 74, 53-62 (1999) Zbl 0941.53014 MR 99m:53100

[FZ2] Florit, L., Zheng, F.: On nonpositively curved Euclidean submanifolds: splitting results, II. J. Reine Angew. Math. 508, 1-15 (1999) Zbl 0911.53005 MR 99m:53101

[FZ3] Florit, L., Zheng, F.: Complete real Kähler Euclidean hypersurfaces are cylinders. Preprint at http://www.preprint.impa.br/Shadows/SERIE_A/2004/274.html

[FZ4] Florit, L., Zheng, F.: Complete real Kähler Euclidean submanifolds in codimension two. Preprint

[Fw] Fwu, C.: Kaehler manifolds isometrically immersed in Euclidean space. J. Differential Geom. 14, 99-103 (1979) Zbl 0441.53052 MR 81h:53057

[H] Hartman, P.: On isometric immersions in Euclidean space of manifolds with non-negative sectional curvatures II. Trans. Amer. Math. Soc. 147, 529-540 (1970) Zbl 0194.22702 MR 41 \#7586

[M] Moore, J.: On isometric immersions of riemannian products. J. Differential Geom. 5, 159168 (1971) Zbl 0213.23804 MR 46 \#6249

[O] Omori, H.: Isometric immersions of Riemannian manifolds. J. Math. Soc. Japan 19, 205214 (1967) Zbl 0154.21501 MR 35 \#6101

[R] Ryan, P.: Kähler manifolds as real hypersurfaces. Duke Math. J. 40, 207-213 (1973) Zbl 0257.53055 MR 49\#1439

[Z] Zheng, F.: Isometric embedding of Kähler manifolds with nonpositive sectional curvature. Math. Ann. 304, 769-784 (1996) Zbl 0874.53040 MR 97e:53127 